\title{
Identification of Tapping Panel Dryness in Hevea brasiliensis Based on Threshold Segmentation Algorithm
}

\author{
Jie Zhang ${ }^{1, \text { a, *, Pan Zhou }}{ }^{2, \mathrm{~b}}$ and Jihong Long ${ }^{2, \mathrm{c}}$ \\ ${ }^{1}$ School of Hainan University, Haikou 570100, China. \\ ${ }^{2}$ School of Hainan University, Haikou 570100, China.

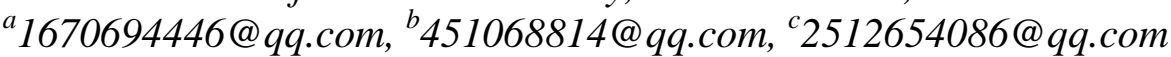

Keywords: image processing; image segmentation; TPD; threshold segmentation algorithm

\begin{abstract}
The tapping panel dryness (TPD) in Hevea brasiliensis is a complex physiological syndrome and seriously affects the yield of the natural latex from rubber tree. The study of TPD is of great significance for improving the yield of latex. The traditional artificial recognition of TPD has a certain deviation due to the high precision of grading workload, so the impact of TPD cannot be paid attention to in time. This paper uses image processing technology in the field of information science to identify the TPD, and uses image segmentation to determine the level of TPD, thus greatly reducing the artificial judgment of the TPD level of misjudgment rate.
\end{abstract}

There are four key steps in the study of TPD recognition, image acquisition, image preprocessing, image segmentation, and determine the level of TPD. Among them, image segmentation is the key to the research of TPD recognition. The gray threshold segmentation algorithm can be used to extract and segment the TPD feature in digital image processing technology, and simulate the experiment through MATLAB. From the results, it can be concluded that in the study of the recognition of TPD in Hevea brasiliensis, the segmentation method based on threshold is simple, the recognition speed is faster and the effect is better. Compared with the traditional artificial recognition method, this method is simpler, more accurate and more timeliness, and thus greatly saves the cost.

\section{Introduction}

In the world, natural rubber is regarded as an indispensable raw material in the industry and an indispensable resource in the development of economy by various countries. As of now, the total annual output of natural rubber accounts for one-third of the production of global rubber. Natural rubber has good quality in elasticity, insulation, abrasion resistance, airtightness and resistance to bending, which is irreplacable,especially for synthetic rubber. With the development of our economy, more and more people tend to select natural rubber. But now the production of natural rubber can not meet the requirement of current development. The severe TPD which is caused by the delayed feedback as well as management on the growth of rubber trees and the state of the bark after tapping, has forced people to stop the production of latex with TPD and reduced the quantities tremendously 
in production. In this case, there is an urgent need to use an efficient, fast, accurate, and economical method to identify TPD and make up for the deficiencies in traditional methods.

The application of image processing technology in the TPD detection and identification will replace the traditional artificial recognition method and greatly reduce the cumbersome process of sampling in the forest rubber tree. It can save the laboratory resources and greatly compress the input of human, financial and material resources. It can not only make up the inaccuracy of the diagnosis of TPD, but also realize fast, accurate and nondestructive diagnosis, and the digital information provides the necessary precondition for the best time to cut rubber tree.

\section{Experimental Procedure}

\subsection{Sample Selection}

The first step of using image processing technology to identify and extract the characteristic parameters of TPD is to collect images. The digital image of the non digital rubber tree under the natural condition is transformed into the digital image which can be recognized by the computer, and then transfers the analog signal to the digital signal, and the recognition function is realized in the computer through processing. The acquisition of images using digital cameras is relatively straightforward, but the images collected are also easily affected by the environment, which makes it more difficult to image processing.

The rubber tree images needed in the experiment were collected from the experimental base, and 276 images were collected at random in the base of the rubber tree. 10 images were selected randomly from the collected pictures for the identification and research test.

There are two principles for selecting rubber tree images in the experiment.

(1) the secant texture of the rubber tree secant is clearer and more complete.

(2) rubber tree secant line is as bright as bark contrast.

The following is a sample of the picture samples of the rubber tree.

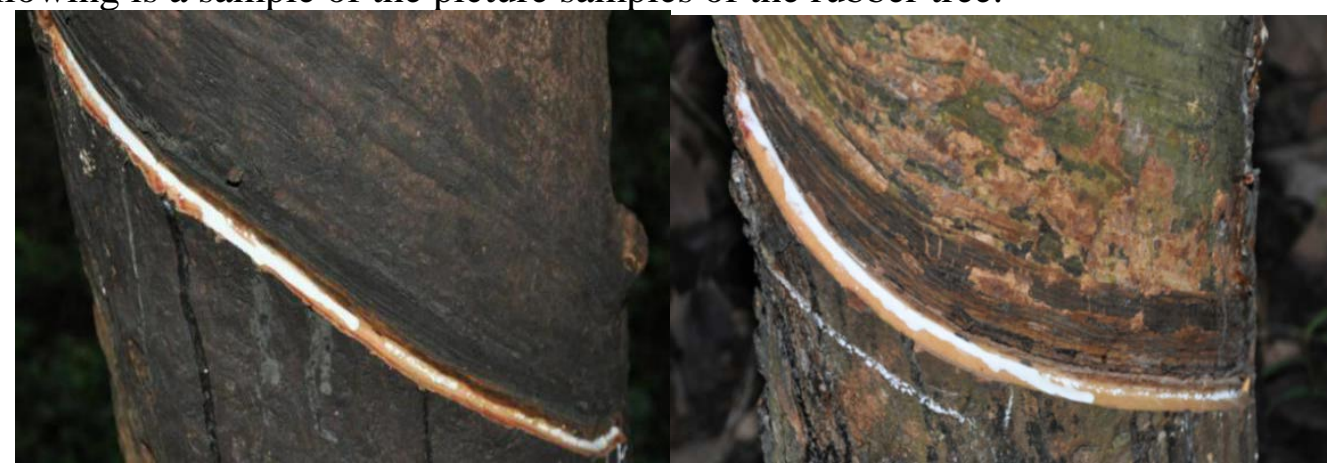

Figure 1. Image samples.

\subsection{Experimental Process}

In the research of TPD recognition based on image processing technology, we use MATLAB to simulate the main steps of image preprocessing and image segmentation recognition. The following is an introduction to the experimental process based on MATLAB platform.

Based on the threshold segmentation algorithm, the flow chart of TPD identification is as follows: 


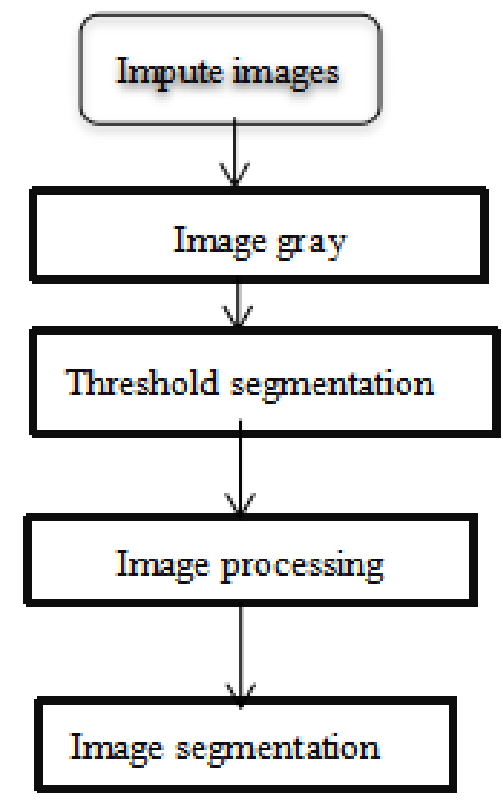

Figure 2. Flow chart

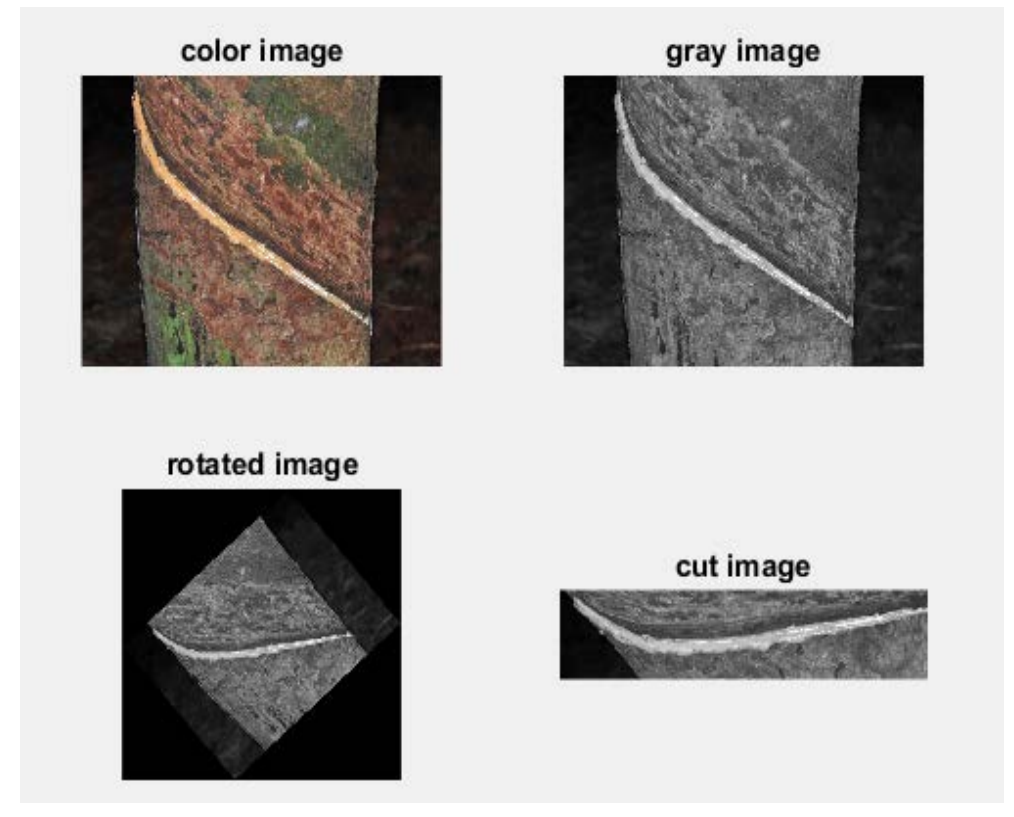

Figure 3. Image processing

In MATLAB, we first input a original color image containing the characteristic of dead skin. By converting the color image into a gray image, we calculate the segmentation threshold according to the iterative method in the threshold segmentation algorithm. The image is divided into two parts of the target background, and then the image is carried out by the expansion corrosion and opening and closing operations in the morphology. In order to reduce the noise, the dead skin and latex of the rubber tree are cut out after the preprocessing of the image rotation and cutting, and the Secant and latex of the rubber tree after segmentation and recognition are output.

The image recognition results of randomly selected several different dead skin grades in image samples are as follows: (a is the secant, $\mathrm{B}$ is the latex of rubber tree). 

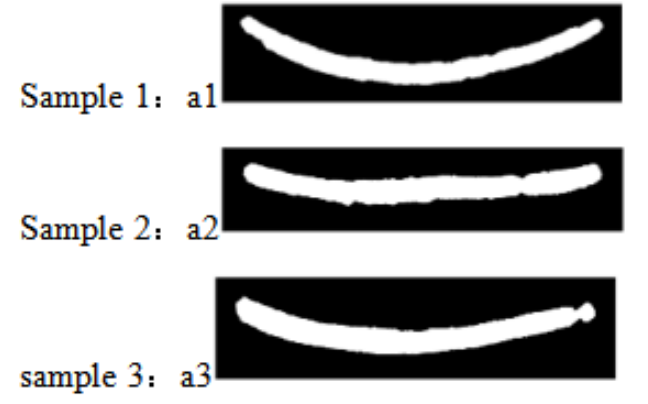

Sample 4: a

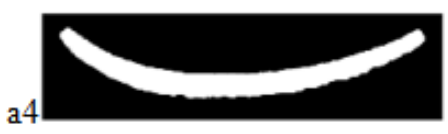

Sample 5:

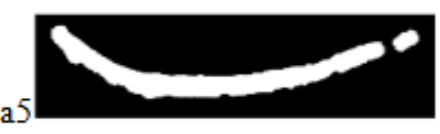

Sample 6:

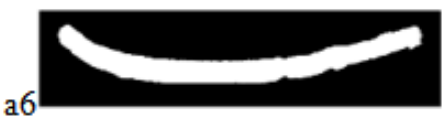

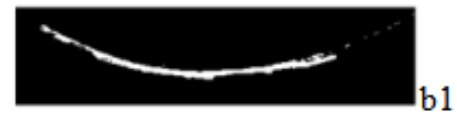
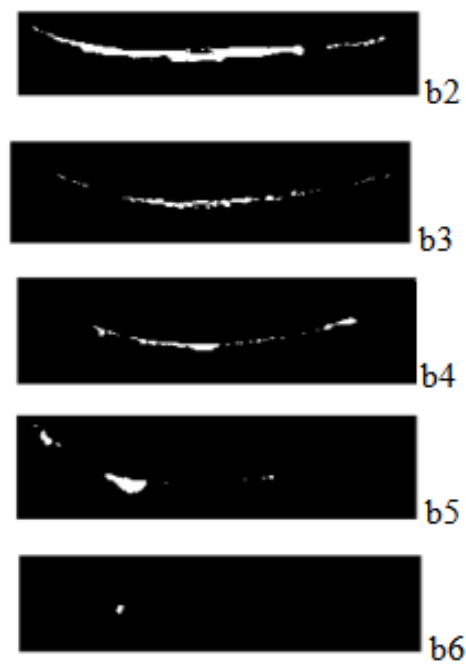

Figure 4. Results.

It can be seen from the dead skin characteristic Secant and latex result map of the dead rubber tree on the top of the rubber tree that the worse the rubber tree dead skin in the image is, the more serious the dead skin disease of the rubber tree is, the level of the dead skin disease of the rubber tree can be determined according to the identified Secant and the area ratio of latex, and the grade of the dead skin disease is from low to high. Sub divided into first, two, three, four, five, six.

Table 1 Treatment of experimental results

\begin{tabular}{ccccc}
\hline Sample & $\begin{array}{c}\text { Secant } \\
\text { graph }\end{array}$ & $\begin{array}{c}\text { The image of } \\
\text { latex }\end{array}$ & $\begin{array}{c}\text { The area ratio of latex / } \\
\text { secant }\end{array}$ & $\begin{array}{c}\text { The level of } \\
\text { TPD(in descending order) }\end{array}$ \\
\hline Sample 1 & a1 & b1 & $1 / 2$ & Level 1 \\
Sample 2 & a2 & b2 & $1 / 3$ & Level 2 \\
Sample 3 & a3 & b3 & $1 / 4$ & Level 3 \\
Sample4 & a4 & b4 & $1 / 5$ & Level 4 \\
Sample 5 & a5 & b5 & $1 / 6$ & Level 5 \\
Sample 6 & a6 & b6 & $1 / 7$ & Level 6 \\
\hline
\end{tabular}

\section{Conclusion}

The recognition results can be obtained that in the research of TPD recognition, the segmentation method based on threshold is simple, fast and effective, which is simpler and more convenient, more accurate and time-consuming than the traditional artificial recognition method, and saves the labor cost greatly. However, this method requires more stringent image samples, such as the collection of complex image samples is poor recognition, so when collecting images, it is necessary to be seriously treated.

\section{References}

[1] Yan Xueqiang, Ye Xiuqing, Liu Jilin. Maximum entropy threshold processing algorithm based on quantized image 
histogram [J]. pattern recognition and artificial intelligence, 1998,11 (3) 352-358.

[2] Chen Zi-kuan,Taoyang,Chen Xin,etal. WaveletBased Adaptive Thresholding Method for Image Segmentation[J].Opt ical Engineering, 2001,40(5)868-874.

[3] Sun Guangling. Image segmentation method based on fuzzy threshold [D]. HeFei University of Technology, 2005.

[4] Sun Shurong. Research and application of entropy based deep sea resource image processing algorithm [D]. Central South University, 2008.

[5] Abutale AS. Automatic thresholding of gray-level pictures using two-dimensional entropies[J]. Computer Vision Graphics Image Process, 1989, 47:22-32.

[6] Liu Yitong, Fu Mengyin. A Fast Infrared Image Segmentation Method Based Two-Dimensional Entropy and Particle Swarm Optimization on Algorithm [J]. Pattern Recognition and Artificial Intelligence, 2008, 21(2): 155-159.

[7] Men Hong, Yu Jiaxue, Qin Lei. Segmentation of Electric Equipment Infrared Image Based on CA and OTSU [J]. Electric Power Automation Equipment, 2011, 31(9): 92-95. 